\section{Response to Walker}

To the Editor: The prenatal reflex DNA test described in our paper $^{1}$ is a method of screening that has advantages over the recall method. ${ }^{2,3}$ In the recall method as described, ${ }^{3}$ a combined test is carried out and women with a risk $\geq 1$ in 150 receive a positive result; they are invited to return for counseling and offered either a second screening test based on a DNA analysis or an invasive diagnostic test-of course, without an obligation to accept either. Walker, in her letter, ${ }^{4}$ does not appear to fully accept the advantages of the reflex method. Reflex DNA screening results in a higher rate of detection ( $95 \%$ vs. $81 \%$ ) of pregnancies with trisomy 21,18 , and 13 and a false-positive rate $(0.02 \%$ vs. $2.4 \%)$ about a hundred times lower than the recall method. Consequently, the reflex method means fewer women with an unaffected pregnancy are given a positive screening result, while a high detection rate is maintained. A quantitative comparison of the two methods in respect of screening for trisomy 21 is given at http://www.wolfson.qmul.ac.uk/ReflexDNAversusRecallDNA.

This shows that in 10,000 pregnancies screened by either the reflex or recall methods, respectively, the following numbers apply: (i) women recalled, 0 vs. 259; (ii) invasive diagnostic tests in unaffected pregnancies, 1.8 vs. 30.3; (iii) trisomy 21 pregnancies detected, 30.3 vs. 25.9; and (iv) odds of being affected given a positive result, 17:1 vs. 1:1. These advantages of the reflex method over the recall method are clear and, contrary to Walker's opinion, involve no extrapolation. It is untenable to suggest that worrying women and their partners with the news of a positive screening result when this is completely unnecessary is good medical practice, or even ethical. Calling women back for another test when this can be avoided causes unnecessary harm and this is always wrong. It is not an issue that is relevant to patient autonomy or choice, which is, of course, important.

Our responses to Walker's four points are as follows. (i) The decision to be screened is an option that women can, of course, discuss with their partners; obtaining consent to reflex DNA screening is no different from obtaining consent to other screening tests. (ii) Notifying women that they have a positive screening result is obviously distressing. If this can be avoided without loss of efficacy it should be done. It is not a legitimate or ethical matter for research. (iii) The duty of health professionals is to offer the most effective and safe tests or interventions that are affordable. This is not paternalistic; it is the expected duty of care. There is no withholding of information, as Walker states in relation to reflex DNA screening, because consent is obtained for the reflex test incorporating all its components. The combined test itself has several components and no one argues that separate consent should be sought for each component (nuchal translucency measurement and two blood measurements). The concept underlying the reflex DNA test is to capture the advantages of a single screening test with several components instead of performing two separate sequential tests. (iv) Avoiding unnecessary harm without loss of efficacy is always a benefit and therefore not a valid research issue.

While there is agreement about using the combined test followed by a DNA test in some women, there is disagreement on how this is done. Walker holds the view that there is merit in women being told that they have a positive combined test result, and then being recalled for counseling. Given that reflex DNA screening can avoid this step entirely, and deliver a test with an improved screening performance, we see no advantage in the recall strategy.

We are puzzled by Walker's view that avoiding the unnecessary reporting of false-positive results is not a selfevident benefit, and puzzled by her questioning the consequential reduced use of clinical resources, which was unambiguously clear to the midwives and clinicians involved. We are also at a loss to understand how Walker seems to lean toward the recall method, when the evidence so strongly favors the reflex method with the added benefit of avoiding needless worry among the women screened.

\section{DISCLOSURE}

N.J.W. is Director of Logical Medical Systems, which produces software for the interpretation of Down syndrome screening tests. The other authors declare no conflict of interest.

Nicholas J. Wald, FRS, FRCP ${ }^{1}$, Wayne J. Huttly, $M S c^{1}$, Jonathan P. Bestwick, $M \mathrm{Sc}^{1}$, Robert Old, $P \mathrm{PD}^{1}$,

Joan K. Morris, PhD ${ }^{1}$, Ray Cheng, MPhil ${ }^{1}$, Joe Aquilina, FRCOG ${ }^{2}$, Elisabeth Peregrine, MRCOG ${ }^{3}$, Devender Roberts, MRCOG ${ }^{4}$ and Zarko Alfirevic, FRCOG ${ }^{4}$

${ }^{1}$ Wolfson Institute of Preventive Medicine, Queen Mary University of London, London, UK; ${ }^{2}$ Maternity Department, Barts Health NHS Trust (Royal London Hospital, Newham General/Whipps Cross Hospitals), London, UK; ${ }^{3}$ Maternity service, Kingston Hospital, London, UK; ${ }^{4}$ Department of Women's and Children's Health, The University of Liverpool, Liverpool, UK. Correspondence: Nicholas J. Wald (n.j.wald@qmul.ac.uk)

\section{REFERENCES}

1. Wald NJ, Huttly WJ, Bestwick JP, et al. Prenatal reflex DNA screening for trisomies 21, 18, and 13. Genet Med; e-pub ahead of print 9 November 2017.

2. Chitty LS, Wright D, Hill M, et al. Uptake, outcome and costs of implementing non-invasive prenatal testing for Down's syndrome into NHS maternity care: prospective cohort study in eight diverse maternity units. BMJ 2016;354:i3426.

3. Public Health England. NHS public health functions agreement 2017-18. Service specification no.16. NHS Fetal Anomaly Screening Programmescreening for Down's, Edwards' and Patau's Syndromes (Trisomy 21, 18 \& 13). https://www.england.nhs.uk/wp-content/uploads/2017/05/serv-spec16.pdf. Accessed 29 November 2017.

4. Walker CL. Response to Wald et al. Genet Med 2018;20:XXX-XXX.

Advance online publication 1 March 2018. doi:10.1038/gim.2017.256 